\title{
Macular perfusion normative data acquired with optical coherence tomography angiography in healthy four-year-old Caucasian children
}

\author{
Dunja Bajtl ${ }^{1}$, Mirjana Bjeloš $23,44^{*}$, Mladen Bušić ${ }^{2,3,4}$, Ana Križanović ${ }^{3,4}$, Leon Marković 3,4 and \\ Biljana Kuzmanović Elabjer 2,3,4
}

\begin{abstract}
Background: The purpose of this cross-sectional study involving healthy emmetropic four-year-old Caucasian children was to provide a macular perfusion normative database acquired with optical coherence tomography angiography (OCTA). One eye of each examinee underwent OCTA imaging. The following parameters were analyzed using AngioTool Image J software: vessels area (VA), vessels density (VD), total number of junctions (TNJ), junctions density $(J D)$, total vessel length (TVL), average vessel length (AVL), total number of endpoints (TNEP), lacunarity ( $L$ ), vessel diameter index (VDI), tortuosity (T) and foveal avascular zone (FAZ). Average central macular thickness (CMT) and average central macular volume (CMV) were measured.

Result: Sixty-two eyes of 62 children of average age 50.4 \pm 3.8 months were examined. VA, VD, and T increased from the inner towards the outer layers of the retina. The intermediate capillary plexus had the highest JD and TNEP and narrowest FAZ. Retinal sexual differentiation was supported with higher values of the retinal VA, VDI and TNEP, and chorioretinal VA, VDI and $L$ in males. The choriocapillaris presented with the highest VD, AVL, and $T$ and the lowest $L$ and TNEP.
\end{abstract}

Conclusion: The study provides the first detailed normative database of the macular vascular network in the youngest uniform cohort of emmetropic four-year-old children.

Keywords: Children, Retina, Perfusion, Angiography, Retinal vessels

\section{Background}

Optical coherence tomography angiography (OCTA) provides in vivo imaging of total retinal perfused microvascular networks as well as the choriocapillaris (CC) and choroid displayed as two-dimensional en face images derived from $3 \mathrm{D}$ volumetric data.

*Correspondence: dr.mbjelos@gmail.com

3 University Eye Department, University Hospital "Sveti Duh", Sveti Duh 64, 10000 Zagreb, Croatia

Full list of author information is available at the end of the article
OCTA segmentation of four vascular plexuses agrees with the vascular distribution obtained by histological analysis. The innermost capillary network, the radial peripapillary capillary plexus (RPCP), is found in the nerve fiber layer. The superficial vascular plexus (SVP) is located within the retinal ganglion cell layer and a superficial portion of the inner plexiform layer (IPL). These two layers are further categorized under the OCTA nomenclature as superficial vascular complex (SVC). The intermediate capillary plexus (ICP) is segmented between the deep portion of IPL and the superficial portion of the original author(s) and the source, provide a link to the Creative Commons licence, and indicate if changes were made. The images or other third party material in this article are included in the article's Creative Commons licence, unless indicated otherwise in a credit line to the material. If material is not included in the article's Creative Commons licence and your intended use is not permitted by statutory regulation or exceeds the permitted use, you will need to obtain permission directly from the copyright holder. To view a copy of this licence, visit http://creativecommons.org/licenses/by/4.0/. The Creative Commons Public Domain Dedication waiver (http://creativeco mmons.org/publicdomain/zero/1.0/) applies to the data made available in this article, unless otherwise stated in a credit line to the data. 
inner nuclear layer (INL). The outermost layer, the deep capillary plexus (DCP) is established at the level of the deep portion of INL. The latter two capillary networks form a deep vascular complex (DVC).

Extensive cytoarchitectonic studies in vivo are thus feasible enabling important information on the intermediate and deep retinal vascular system that fluorescein angiography (FA) and indocyanine green (ICG) imaging cannot provide. The OCTA has amplified the analysis of microvasculature of $\mathrm{CC}$ as dye-based imaging techniques have poor lateral resolution. Data on the macular vascular network in children are limited [1-3], and knowledge on the development of human fovea is mostly gathered via histological studies. As a non-contact, non-invasive tool it can be implemented in cooperative children, in particular when frequent monitoring is needed.

Thus, we performed an analysis of macular microvascular architecture and ocular biometry in children at a onetime point to expand and comprehend the knowledge on macular vascular developmental trajectory during the process of foveal maturation and eye emmetropization.

\section{Methods}

\section{Study description and oversight}

This cross-sectional study was conducted at the University Eye Department, University Hospital "Sveti Duh", Zagreb, Croatia, between January 2019 and March 2020.

\section{Study participants}

Healthy participants of the Croatian Preventive Program for Early Amblyopia Detection in four-year-old children were recruited in the study [4].

Gender, date of birth, pregnancy, and developmental data for any systemic and eye diseases were obtained from legal guardians. All examinees were tested for visual acuity without correction at near $(40 \mathrm{~cm})$ and distance $(3 \mathrm{~m})$ using Lea symbols ${ }^{\circledR}$ inline test, binocularly and monocularly adhering to Zagreb Amblyopia Preschool Screening protocol [5]. One eye randomly chosen per examinee underwent OCTA imaging using SPECTRALIS ${ }^{\circledR}$ OCT Angiography (HRA + OCT Spectralis, Heidelberg Engineering, Heidelberg, Germany) followed by axial length measurements acquired with IOLMaster $^{\circledR} 700$ (Carl Zeiss Meditec AG, Jena, Germany). All measurements were performed by a single experienced operator (D. B.). The imaging was performed in a dark room, with the other eye patched.

Inclusion criteria were defined as follows: (1) visual acuity without correction for distance and for near tested binocularly and monocularly $\leq 0.1 \log$ Mar, (2) OCTA quality index $\mathrm{Q} \geq 30$.

Exclusion criteria were: (1) presence of any known ocular and/or systemic diseases, malformations, or previous intraocular surgery; (2) inadequate fixation on IOLMaster visible on cross-section scan.

\section{OCTA imaging}

OCTA imaging was performed using an A-scan pattern of $10 \times 10^{\circ}(\sim 2.9 \times 2.9 \mathrm{~mm})$ containing $512 \mathrm{~A}$-scans $\times 512$ B-scans. A high-resolution scan of $5.7 \mu \mathrm{m} /$ pixel was applied.

Vascular layers were segmented automatically: RPCP, SVP, ICP, DCP, CC, and choroid. Retina slab represented the image of all retinal vessels and Full referred to the image of all chorioretinal vessels.

Skeletonized images with $\mathrm{Q} \geq 30 \mathrm{~dB}$ were further calibrated using 162 pixels per $1 \mathrm{~mm}$ to analyze vascular parameters in $\sim 8.4 \mathrm{~mm}^{2}$ of explanted area (EA) using AngioTool Image J software (version 0.6a, 02.18.14) (Fig. 1). AngioTool is an open-source software provided by the National Cancer Institute (National Institutes of Health $^{\circledR}$, Bethesda, MD, USA) for vessel parameters analysis: vessels area (area of the segmented vessels inside the explant area, VA), vessels density (percentage of the area of the segmented vessels inside the explant area, VD), total number of junctions (total number of vessel junctions inside the explant area, TNJ), junctions density (number of vessel junctions normalized per unit area, JD), total vessel length (sum of Euclidean distances between the pixels of all the vessels inside the explant area, TVL), average vessel length (mean length of all the vessels inside the explant area, AVL), total number of endpoints (number of open-ended vessel segments, TNEP) and lacunarity (mean lacunarity overall size boxes, L) [6]. A vessel is defined as a segment between a branching point and an endpoint or two branching points [6].

Integrated TruTrack@ Active Eye Tracking corrected displacements of retinal layers due to the reacquisition of images at the correct retinal location, motion, and blinking artifacts [7].

Average central macular thickness (CMT), average central macular volume (CMV), foveolar thickness (FoT), average parafoveolar temporal macular thickness (PTT), average parafoveolar temporal macular volume (PTV), average parafoveolar nasal macular thickness (PNT), average parafoveolar nasal macular volume (PNV), average parafoveolar superior macular thickness (PST), average parafoveolar superior macular volume (PSV), average parafoveolar inferior macular thickness (PIT) and average parafoveolar inferior macular volume (PIV) were obtained automatically using integrated macular thickness map mode.

Foveal avascular zone (FAZ) delineation was performed by a single experienced operator (D. B.) as presented by Zhao et al., for each vascular layer separately, using Adobe Photoshop CC (version 2019 20.0) [8]. 


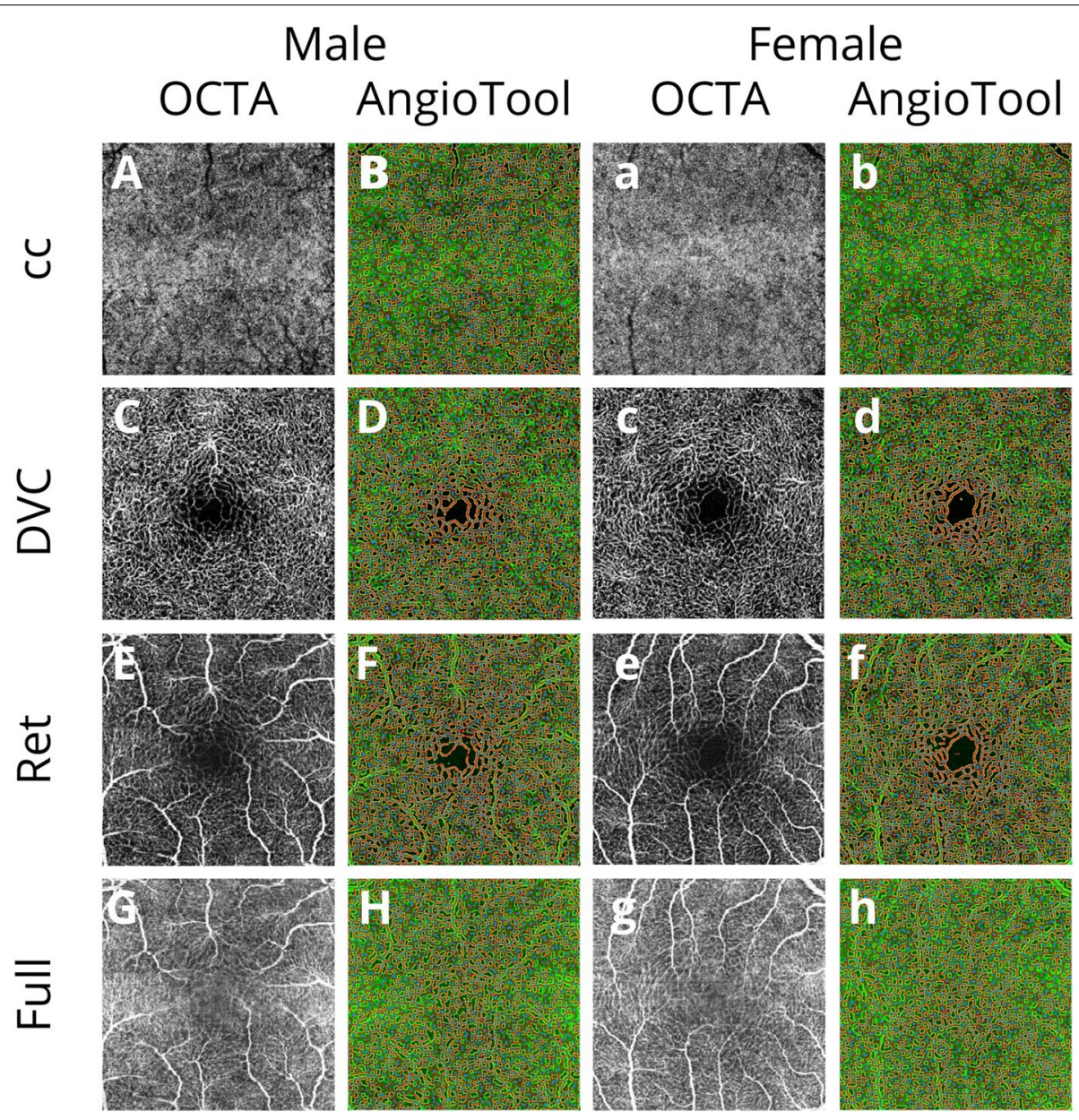

Fig. 1 OCTA en-face images (image quality $Q=43$ ) showing binary skeletal structure in the left column $\mathbf{A}, \mathbf{C}, \mathbf{E}, \mathbf{G}, \mathbf{a}, \mathbf{c}, \mathbf{e}, \mathbf{g}$, and skeletonized images with overlay of the AngioTool output in the corresponding right column $\mathbf{B}, \mathbf{D}, \mathbf{F}, \mathbf{H}, \mathbf{b}, \mathbf{d}, \mathbf{f}, \mathbf{h}$, of a male (left column) and a female examinee (right column). The skeletons are outlined in red, vessels are presented yellow, branching points are indicated in blue. Calculated vascular parameters are presented in the Table 1

Mathematical delineation of FAZ area in $\mathrm{mm}^{2}$ is presented in the equation:

$$
F A Z=\frac{N P}{N P M \times M}
$$

where $N P$ corresponds to the number of pixels enclosed by FAZ area, NPM to the number of pixels per $\mathrm{mm}^{2}$, and $M$ refers to the magnification.

Vessel diameter index (VDI) [9], and tortuosity (T) were calculated according to the following formulas:

$$
\begin{aligned}
& V D I=1000 x \frac{V A}{T V L} \\
& T=\frac{(T N J+T N E P-1) \times A V L}{T V L}
\end{aligned}
$$

\section{Biometric eye components measurement}

All examinees underwent analysis of the axial length $(\mathrm{AL})$, anterior chamber depth (ACD), lens thickness (LT), central corneal thickness (CCT), corneal curvature in the flattest (K1) and steepest (K2) meridian, horizontal white-to-white (WTW) utilizing IOLMaster ${ }^{\circledR} 700$ biometer.

The power of the Acrysof IQ Monofocal SN60WF (Alcon Laboratories Inc., Fort Worth, Texas, USA), with A-constant 118.7 was calculated using the SRK-T formula [10].

\section{Statistical analysis}

The primary study outcome was the analysis of vessel parameters VA, VD, TNJ, JD, TVL, AVL, TNEP, L, VDI, and $\mathrm{T}$ and metrics on the macular thickness and volume 
CMT, CMV, FoT, PTT, PTV, PNT, PNV, PST, PSV, PIT, and PIV. Secondary outcomes included assessments of biometric eye components AL, ACD, LT, CCT, K1, K2, WTW, and intraocular lens power.

Categorical data were presented with absolute and relative frequencies, while numerical data were presented with arithmetic mean and standard deviation if distributed within parameters of normal Gaussian distribution. Numerical data that did not fit the normal distribution were presented with median and interquartile range.

Differences between the two independent sets of numerical data were tested with the nonparametric Mann-Whitney U test, while differences between more than two dependent sets of numerical data were tested with the nonparametric Friedman test and Conover posthoc test. The sexual differentiation for each of the parameters of primary and secondary outcomes. Statistical analysis was performed with MedCalc (19.1.3, MedCalc Software bv) and IBM SPSS Statistics (release 24.0.0.0) software tools, with statistical significance defined as $\alpha=0.05$, where all $P$ values were two-tailed.

\section{Results}

\section{Study participants}

The study examined 62 eyes of 62 children of average age $50.4 \pm 3.8$ (mean $\pm \mathrm{SD}$ ) months (range 48 to 59 months of age). There were 30 (48.4\%) right eyes, and 32 (51.6\%) left ( $\chi^{2}$ test, $P=0.86$ ). Twenty-nine $(46.8 \%)$ children were male and thirty-three $(53.2 \%)$ were female $\left(x^{2}\right.$ test, $P=0.72$ ). EA was equal across all vascular layers. Statistical difference was not found (Friedman test; $P=0.12$ ).

\section{Primary outcome measures}

Table 1 presents normative data of sexual differentiation of examined vascular parameters VA, VD, TNJ, JD, TVL, AVL, TNEP, L, VDI, T, FAZ area, macular thickness, and volume (Mann-Whitney $U$ test, $P<0.05$ ). The distribution of the vascular parameters VA, VD, TNJ, JD, TVL, AVL, TNEP, L, VDI, T, and FAZ area across the layers is presented in Additional file 1: Table S1. The macular thickness and volume distribution are presented in Additional file 1: Table S2. Heterogeneity of vascular layers for the vascular parameters VA, VD, TNJ, JD, TVL, AVL, TNEP, L, VDI, T, and FAZ area is analyzed in Additional file 1: Table S3 (Conover post-hoc test, $P<0.05$ ).

\section{Secondary outcome measures}

The analyzed biometric eye components $\mathrm{AL}, \mathrm{ACD}, \mathrm{LT}$, CCT, K1, K2, WTW, and refractive eye power (Student's t-test, $P<0.05)$ (Table 2) did not demonstrate sexual dimorphism in four-year-old Caucasian children (Additional file 1: Table S4).

\section{Discussion}

This study provides the first detailed quantitative delineation of the macular vascular network in the youngest uniform cohort of emmetropic four-year-old children of Caucasian origin.

Uniform Explant area (Friedman test; $P=0.12$ ) across layers enabled reliable further statistical analysis of the measured primary endpoints.

The morphological development of the macula takes place predominantly during the first 5 years of life, thus demonstrating different timeframes of layers development. The development of the inner retinal layers is completed by 5 months [11], while adult human cone density is reached between 4 and 6 years of age [12]. A full adult architecture is reached by 10 years of age [9]. This is the first study revealing that retinal VA, VD, and $T$ increased from the inner towards the outer layers of the retina with SVP and ICP demonstrating no difference in VA and VD (Additional file 1: Tables S1, S3). This is consistent with the functional distribution of the regions of the highest oxygen demands and light absorption as follows: inner plexiform layer, outer plexiform layer, and photoreceptor layer.

On the contrary, in adult human donor eye, capillary density was the highest in networks supplying the IPL, namely SVP and ICP, and lowest in DVP [13]. Thus, it is possible that the microvascular model observed in this study extends until the normal aging process commences. The characteristic pattern of the normal aging affecting adults after 35 years of age is presented with decreased retinal tissue perfusion in DVP as the primary event. This is followed by further changes in volumetric vessel density in DVP and SVP in opposite directions, demonstrating a decrease and increase, respectively [14].

We found significantly larger vessel areas in boys in the DVC, retina layer and the entire chorioretina compared to girls (Table 1). This poses an important question: whether boys mature earlier and similarity between genders evolves later in the developmental process? The alternative is that this structural dimension of sexual dimorphism is finite and plays a protective role during aging as macular perfusion decreases more rapidly in males [9]. The latter scenario could be reinforced with observed variations in the foveal capillary-free area being larger in healthy adult females [1, 15-17].

Retinal sexual differentiation was further supported with higher values of the retinal TNEP and chorioretinal lacunarity in males compared to their female counterparts (Table 1). Lacunarity discloses a more heterogeneous spatial arrangement of the vessels in males. 
Table 1 Sexual differentiation of examined vascular parameters

\begin{tabular}{|c|c|c|c|c|c|c|c|c|}
\hline \multirow{2}{*}{$\begin{array}{l}\text { Variable } \\
\text { (measurement unit) }\end{array}$} & \multirow[t]{2}{*}{ Layer } & \multicolumn{3}{|l|}{ Male } & \multicolumn{3}{|l|}{ Female } & \multirow[t]{2}{*}{$P$ Value ${ }^{*}$} \\
\hline & & Mean & 25th percentile & 75th percentile & Mean & 25th percentile & 75th percentile & \\
\hline \multirow[t]{10}{*}{$\mathrm{EA}\left(\mathrm{mm}^{2}\right)$} & $\mathrm{RPCP}$ & 8.38 & 8.38 & 8.38 & 8.38 & 8.38 & 8.38 & 0.64 \\
\hline & SVP & 8.38 & 8.38 & 8.38 & 8.38 & 8.38 & 8.38 & 0.16 \\
\hline & $I C P$ & 8.38 & 8.38 & 8.38 & 8.38 & 8.38 & 8.38 & 0.62 \\
\hline & DCP & 8.38 & 8.38 & 8.38 & 8.38 & 8.38 & 8.38 & 0.08 \\
\hline & $C C$ & 8.38 & 8.38 & 8.38 & 8.38 & 8.38 & 8.38 & 0.09 \\
\hline & Choroid & 8.38 & 8.38 & 8.38 & 8.38 & 8.38 & 8.38 & 0.40 \\
\hline & SVC & 8.38 & 8.38 & 8.38 & 8.38 & 8.38 & 8.38 & 0.07 \\
\hline & DVC & 8.38 & 8.38 & 8.38 & 8.38 & 8.38 & 8.38 & 0.23 \\
\hline & Ret & 8.38 & 8.38 & 8.38 & 8.38 & 8.38 & 8.38 & 0.56 \\
\hline & Full & 8.38 & 8.38 & 8.38 & 8.38 & 8.38 & 8.38 & 0.62 \\
\hline \multirow[t]{10}{*}{$\mathrm{VA}\left(\mathrm{mm}^{2}\right)$} & $\mathrm{RPCP}$ & 3.44 & 2.89 & 3.94 & 3.32 & 2.64 & 3.65 & 0.38 \\
\hline & SVP & 5.46 & 5.27 & 5.69 & 5.43 & 5.08 & 5.61 & 0.30 \\
\hline & $I C P$ & 5.53 & 5.37 & 5.63 & 5.45 & 5.17 & 5.60 & 0.31 \\
\hline & DCP & 5.70 & 5.55 & 5.85 & 5.60 & 5.45 & 5.78 & 0.16 \\
\hline & CC & 6.69 & 6.45 & 6.80 & 6.61 & 6.50 & 6.78 & 0.97 \\
\hline & Choroid & 5.46 & 5.29 & 5.54 & 5.57 & 5.38 & 5.72 & 0.05 \\
\hline & SVC & 5.04 & 4.87 & 5.33 & 4.97 & 4.52 & 5.20 & 0.15 \\
\hline & DVC & 5.93 & 5.78 & 6.09 & 5.81 & 5.62 & 6.02 & 0.08 \\
\hline & Ret & 5.43 & 5.14 & 5.62 & 5.20 & 4.94 & 5.49 & 0.03 \\
\hline & Full & 5.72 & 5.53 & 5.78 & 5.51 & 5.37 & 5.74 & 0.04 \\
\hline \multirow[t]{10}{*}{ VD (\%) } & $\mathrm{RPCP}$ & 41.07 & 34.54 & 46.98 & 39.64 & 31.47 & 43.55 & 0.38 \\
\hline & SVP & 65.12 & 62.91 & 67.91 & 64.84 & 60.59 & 66.95 & 0.31 \\
\hline & $I C P$ & 66.02 & 64.08 & 67.19 & 64.98 & 61.72 & 66.87 & 0.31 \\
\hline & DCP & 68.02 & 66.20 & 69.84 & 66.81 & 65.10 & 69.03 & 0.16 \\
\hline & $C C$ & 79.86 & 76.98 & 81.13 & 78.87 & 77.61 & 80.88 & 0.97 \\
\hline & Choroid & 65.17 & 63.09 & 66.09 & 66.53 & 64.27 & 68.21 & 0.05 \\
\hline & SVC & 60.14 & 58.14 & 63.56 & 59.29 & 53.97 & 62.12 & 0.16 \\
\hline & DVC & 70.76 & 68.93 & 72.67 & 69.38 & 67.06 & 71.87 & 0.08 \\
\hline & Ret & 64.82 & 61.37 & 67.10 & 62.04 & 59.01 & 65.52 & 0.03 \\
\hline & Full & 68.32 & 66.04 & 68.95 & 65.76 & 64.13 & 68.48 & 0.04 \\
\hline \multirow[t]{10}{*}{ TNJ } & $\mathrm{RPCP}$ & 1196.00 & 948.50 & 1391.50 & 1114.00 & 831.50 & 1235.00 & 0.23 \\
\hline & SVP & 1530.00 & 1486.50 & 1581.50 & 1516.00 & 1471.50 & 1564.00 & 0.33 \\
\hline & $I C P$ & 1763.00 & 1715.00 & 1802.50 & 1757.00 & 1695.50 & 1789.00 & 0.45 \\
\hline & DCP & 1696.00 & 1653.50 & 1737.50 & 1692.00 & 1636.50 & 1737.00 & 0.69 \\
\hline & CC & 1628.00 & 1596.50 & 1721.00 & 1658.00 & 1610.50 & 1742.00 & 0.23 \\
\hline & Choroid & 1625.00 & 1594.00 & 1655.50 & 1602.00 & 1551.50 & 1674.00 & 0.59 \\
\hline & SVC & 1494.00 & 1432.50 & 1589.50 & 1496.00 & 1381.00 & 1573.00 & 0.37 \\
\hline & DVC & 1781.00 & 1733.00 & 1834.50 & 1762.00 & 1704.50 & 1807.00 & 0.11 \\
\hline & Ret & 1781.00 & 1743.50 & 1858.00 & 1781.00 & 1699.50 & 1813.50 & 0.20 \\
\hline & Full & 1910.00 & 1868.00 & 1930.50 & 1910.00 & 1875.50 & 1930.50 & 0.75 \\
\hline \multirow[t]{10}{*}{$\mathrm{JD}\left(\mathrm{mm}^{2}\right)$} & $\mathrm{RPCP}$ & 142.73 & 113.20 & 166.04 & 132.95 & 99.22 & 147.37 & 0.23 \\
\hline & SVP & 182.56 & 177.39 & 188.73 & 180.91 & 175.57 & 186.65 & 0.33 \\
\hline & $I C P$ & 210.38 & 204.65 & 215.09 & 209.68 & 202.33 & 213.47 & 0.46 \\
\hline & DCP & 202.38 & 197.31 & 207.33 & 201.90 & 195.28 & 207.27 & 0.70 \\
\hline & CC & 194.28 & 190.50 & 205.34 & 197.82 & 192.19 & 207.86 & 0.23 \\
\hline & Choroid & 193.88 & 190.21 & 197.54 & 191.15 & 185.15 & 199.76 & 0.59 \\
\hline & SVC & 178.31 & 170.97 & 189.70 & 178.49 & 164.78 & 187.71 & 0.39 \\
\hline & DVC & 212.51 & 206.80 & 218.89 & 210.25 & 203.43 & 215.62 & 0.11 \\
\hline & Ret & 212.60 & 208.03 & 221.72 & 212.51 & 202.81 & 216.42 & 0.20 \\
\hline & Full & 227.89 & 222.92 & 230.37 & 227.95 & 223.78 & 230.39 & 0.76 \\
\hline
\end{tabular}


Table 1 (continued)

\begin{tabular}{|c|c|c|c|c|c|c|c|c|}
\hline \multirow{2}{*}{$\begin{array}{l}\text { Variable } \\
\text { (measurement unit) }\end{array}$} & \multirow[t]{2}{*}{ Layer } & \multicolumn{3}{|l|}{ Male } & \multicolumn{3}{|l|}{ Female } & \multirow[t]{2}{*}{$P$ Value ${ }^{*}$} \\
\hline & & Mean & 25th percentile & 75th percentile & Mean & 25th percentile & 75th percentile & \\
\hline \multirow[t]{10}{*}{ TVL (mm) } & $\mathrm{RPCP}$ & 125.30 & 105.90 & 137.76 & 117.72 & 97.55 & 128.03 & 0.22 \\
\hline & SVP & 152.15 & 148.52 & 154.93 & 151.45 & 148.23 & 153.68 & 0.31 \\
\hline & $I C P$ & 163.42 & 161.20 & 164.87 & 163.64 & 159.22 & 164.91 & 0.64 \\
\hline & DCP & 159.53 & 156.21 & 161.72 & 159.00 & 155.86 & 161.49 & 0.48 \\
\hline & $\mathrm{CC}$ & 159.86 & 158.32 & 163.56 & 162.00 & 159.23 & 164.14 & 0.29 \\
\hline & Choroid & 157.13 & 154.26 & 158.96 & 156.45 & 153.39 & 159.96 & 0.91 \\
\hline & SVC & 149.66 & 146.34 & 154.77 & 149.39 & 141.86 & 152.77 & 0.35 \\
\hline & DVC & 164.29 & 161.98 & 166.37 & 163.34 & 161.11 & 165.04 & 0.10 \\
\hline & Ret & 164.14 & 161.20 & 167.02 & 163.44 & 159.66 & 165.66 & 0.14 \\
\hline & Full & 170.18 & 168.52 & 171.23 & 169.68 & 166.94 & 170.94 & 0.29 \\
\hline \multirow[t]{10}{*}{$\mathrm{AVL}(\mathrm{mm})$} & $\mathrm{RPCP}$ & 0.73 & 0.40 & 1.01 & 0.56 & 0.30 & 0.76 & 0.17 \\
\hline & SVP & 8.71 & 7.07 & 13.50 & 7.87 & 5.46 & 10.85 & 0.13 \\
\hline & $I C P$ & 10.11 & 7.04 & 16.57 & 9.11 & 7.09 & 13.54 & 0.54 \\
\hline & DCP & 11.14 & 9.75 & 14.53 & 11.46 & 9.89 & 14.40 & 0.85 \\
\hline & $C C$ & 55.44 & 39.86 & 82.59 & 78.95 & 46.70 & 120.32 & 0.57 \\
\hline & Choroid & 11.89 & 7.76 & 21.15 & 15.42 & 9.48 & 26.76 & 0.18 \\
\hline & SVC & 7.57 & 4.60 & 9.56 & 6.36 & 3.89 & 7.99 & 0.11 \\
\hline & DVC & 27.87 & 20.50 & 40.62 & 20.10 & 11.57 & 32.92 & 0.01 \\
\hline & Ret & 7.20 & 5.21 & 12.51 & 5.89 & 4.70 & 9.14 & 0.13 \\
\hline & Full & 28.09 & 16.19 & 49.45 & 18.63 & 13.44 & 31.18 & 0.04 \\
\hline \multirow[t]{10}{*}{ TNEP } & $\mathrm{RPCP}$ & 734.00 & 598.50 & 878.00 & 778.00 & 655.50 & 931.00 & 0.36 \\
\hline & SVP & 143.00 & 124.00 & 180.00 & 154.00 & 134.50 & 196.00 & 0.13 \\
\hline & $I C P$ & 148.00 & 131.50 & 179.00 & 162.00 & 126.50 & 199.00 & 0.41 \\
\hline & DCP & 128.00 & 106.50 & 145.00 & 131.00 & 111.50 & 151.50 & 0.47 \\
\hline & $C C$ & 59.00 & 53.00 & 75.00 & 64.00 & 55.00 & 74.50 & 0.86 \\
\hline & Choroid & 206.00 & 174.50 & 236.50 & 181.00 & 152.50 & 222.00 & 0.08 \\
\hline & SVC & 180.00 & 149.50 & 237.50 & 196.00 & 171.00 & 304.00 & 0.08 \\
\hline & DVC & 93.00 & 77.50 & 105.00 & 93.00 & 81.50 & 129.00 & 0.22 \\
\hline & Ret & 171.00 & 144.00 & 219.00 & 204.00 & 165.50 & 276.00 & 0.03 \\
\hline & Full & 133.00 & 109.50 & 167.00 & 162.00 & 126.50 & 198.50 & 0.07 \\
\hline \multirow[t]{10}{*}{$L^{* *}$} & $\mathrm{RPCP}$ & 0.11 & 0.08 & 0.14 & 0.14 & 0.09 & 0.19 & 0.05 \\
\hline & SVP & 0.03 & 0.02 & 0.04 & 0.03 & 0.03 & 0.04 & 0.46 \\
\hline & $I C P$ & 0.02 & 0.01 & 0.02 & 0.02 & 0.02 & 0.03 & 0.62 \\
\hline & $\mathrm{DCP}$ & 0.03 & 0.03 & 0.03 & 0.03 & 0.03 & 0.04 & 0.45 \\
\hline & $C C$ & 0.01 & 0.01 & 0.01 & 0.01 & 0.01 & 0.01 & 0.98 \\
\hline & Choroid & 0.01 & 0.01 & 0.01 & 0.01 & 0.01 & 0.01 & 0.18 \\
\hline & SVC & 0.04 & 0.03 & 0.05 & 0.04 & 0.04 & 0.05 & 0.21 \\
\hline & DVC & 0.02 & 0.02 & 0.02 & 0.02 & 0.02 & 0.03 & 0.19 \\
\hline & Ret & 0.02 & 0.02 & 0.02 & 0.02 & 0.02 & 0.03 & 0.10 \\
\hline & Full & 0.0107 & 0.0093 & 0.0126 & 0.0122 & 0.0111 & 0.0132 & 0.026 \\
\hline \multirow[t]{7}{*}{ FAZ area $\left(\mathrm{mm}^{2}\right)$} & RPCP & 1.13 & 0.72 & 1.42 & 1.27 & 0.93 & 1.61 & 0.10 \\
\hline & SVP & 0.55 & 0.36 & 0.67 & 0.52 & 0.41 & 0.64 & 0.99 \\
\hline & $I C P$ & 0.22 & 0.13 & 0.29 & 0.23 & 0.12 & 0.32 & 0.76 \\
\hline & DCP & 0.52 & 0.43 & 0.59 & 0.53 & 0.38 & 0.65 & 0.64 \\
\hline & SVC & 0.62 & 0.44 & 0.78 & 0.65 & 0.45 & 0.75 & 0.86 \\
\hline & DVC & 0.26 & 0.15 & 0.35 & 0.30 & 0.17 & 0.36 & 0.48 \\
\hline & Ret & 0.28 & 0.17 & 0.33 & 0.31 & 0.17 & 0.39 & 0.36 \\
\hline
\end{tabular}


Table 1 (continued)

\begin{tabular}{|c|c|c|c|c|c|c|c|c|}
\hline \multirow{2}{*}{$\begin{array}{l}\text { Variable } \\
\text { (measurement unit) }\end{array}$} & \multirow[t]{2}{*}{ Layer } & \multicolumn{3}{|l|}{ Male } & \multicolumn{3}{|l|}{ Female } & \multirow[t]{2}{*}{$P$ Value* } \\
\hline & & Mean & 25th percentile & 75th percentile & Mean & 25th percentile & 75th percentile & \\
\hline \multirow[t]{10}{*}{ VDI $(\mu \mathrm{m})$} & RPCP & 27.62 & 26.90 & 28.72 & 27.74 & 26.37 & 29.05 & 0.84 \\
\hline & SVP & 35.90 & 34.56 & 36.95 & 35.68 & 34.03 & 36.57 & 0.41 \\
\hline & $I C P$ & 33.66 & 33.00 & 34.22 & 33.28 & 32.62 & 34.15 & 0.31 \\
\hline & $\mathrm{DCP}$ & 35.66 & 34.44 & 37.33 & 35.21 & 34.18 & 36.19 & 0.27 \\
\hline & $\mathrm{CC}$ & 41.99 & 39.20 & 42.82 & 41.50 & 39.63 & 42.28 & 0.79 \\
\hline & Choroid & 34.60 & 33.69 & 35.44 & 35.62 & 34.43 & 36.05 & 0.01 \\
\hline & SVC & 33.75 & 32.72 & 34.61 & 33.08 & 31.60 & 34.23 & 0.14 \\
\hline & DVC & 35.91 & 35.01 & 37.48 & 35.39 & 34.66 & 36.67 & 0.19 \\
\hline & Ret & 32.68 & 31.13 & 34.22 & 31.82 & 31.09 & 33.02 & 0.05 \\
\hline & Full & 33.42 & 32.82 & 33.99 & 32.47 & 31.91 & 33.66 & 0.04 \\
\hline \multirow[t]{10}{*}{ T } & $\mathrm{RPCP}$ & 11.14 & 6.82 & 14.84 & 8.98 & 5.57 & 11.54 & 0.15 \\
\hline & SVP & 96.56 & 78.10 & 148.27 & 85.68 & 60.94 & 119.11 & 0.11 \\
\hline & $\mathrm{ICP}$ & 114.13 & 83.46 & 193.42 & 106.17 & 83.25 & 157.75 & 0.49 \\
\hline & DCP & 128.86 & 111.69 & 168.55 & 131.57 & 113.74 & 163.36 & 0.92 \\
\hline & $C C$ & 612.67 & 421.25 & 925.50 & 835.50 & 499.96 & 1251.00 & 0.48 \\
\hline & Choroid & 139.77 & 90.53 & 240.73 & 173.00 & 108.25 & 309.33 & 0.20 \\
\hline & SVC & 80.90 & 53.69 & 105.72 & 71.50 & 44.88 & 89.21 & 0.15 \\
\hline & DVC & 323.50 & 232.81 & 453.88 & 224.75 & 129.56 & 365.90 & 0.01 \\
\hline & Ret & 87.26 & 63.10 & 148.11 & 71.36 & 58.17 & 110.36 & 0.15 \\
\hline & Full & 341.67 & 196.35 & 568.79 & 229.67 & 161.56 & 370.33 & 0.04 \\
\hline \multirow[t]{6}{*}{ Macular thickness $(\mu \mathrm{m})$} & CMT & 252.00 & 239.00 & 267.50 & 256.00 & 240.50 & 265.00 & 0.76 \\
\hline & FoT & 204.00 & 197.50 & 213.00 & 207.00 & 199.50 & 216.50 & 0.32 \\
\hline & PTT & 328.00 & 314.00 & 334.50 & 324.00 & 319.00 & 333.00 & 0.89 \\
\hline & PNT & 335.00 & 324.50 & 347.00 & 336.00 & 327.00 & 344.50 & 0.78 \\
\hline & PST & 338.00 & 326.50 & 348.00 & 339.00 & 332.50 & 347.50 & 0.53 \\
\hline & PIT & 339.00 & 325.50 & 344.00 & 334.00 & 327.50 & 343.00 & 0.52 \\
\hline \multirow[t]{5}{*}{ Macular volume $(\mu \mathrm{l})$} & CMV & 0.20 & 0.19 & 0.21 & 0.20 & 0.19 & 0.21 & 0.94 \\
\hline & PTV & 0.52 & 0.49 & 0.52 & 0.51 & 0.50 & 0.52 & 0.69 \\
\hline & PNV & 0.53 & 0.51 & 0.54 & 0.53 & 0.51 & 0.54 & 0.71 \\
\hline & PSV & 0.53 & 0.51 & 0.55 & 0.53 & 0.52 & 0.55 & 0.46 \\
\hline & PIV & 0.53 & 0.51 & 0.54 & 0.53 & 0.51 & 0.54 & 0.66 \\
\hline
\end{tabular}

EA explant area, VA vessels area, VD vessels density, TNJ total number of junctions, JD junctions density, TVL total vessel length, $A V L$ average vessel length, TNEP total number of endpoints, $L$ mean lacunarity, $F A Z$ foveal avascular zone, VD/ vessel diameter index, $T$ tortuosity, $R P C P$ radial peripapillary capillary plexus, SVP superficial vascular plexus, ICP intermediate vascular plexus, DCP deep capillary plexus, SVC superficial vascular complex, DVC deep vascular complex, CC choriocapillaris, Ret retina, Full chorioretina, CMT central macular thickness, CMV central macular volume, FoT foveolar thickness, PTT parafoveal temporal thickness, PTV parafoveal temporal volume, $P N T$ parafoveal nasal thickness, PNV parafoveal nasal volume, $P S T$ parafoveal superior thickness, PSV parafoveal superior volume, PIT parafoveal inferior thickness, PIV parafoveal inferior volume

*Statistical significance was measured using Mann-Whitney U Test. Bolded values denote statistical significance at the level $P<0.05$

**The chorioretinal lacunarity value is presented with four decimal places due to better observation of statistically significant differences

We speculate if this component could be the coupled to complement the process of a more rapid decline of perfusion in males [15].

Sex-based differences in neuroretinal function presume a causal relationship to estradiol (17ß-estradiol, E2) levels [18]. Increased retinal thickness in male rat retina is influenced by prenatal and especially neonatal testosterone surge, not evidenced in females, and its local conversion to estradiol [19]. E2 induces angiogenesis by enhancing the VEGF expression, vasodilation, and thus increased blood flow [20]. The inner nuclear layer, the only layer supported by two capillary networks, ICP and DCP, is reported to be the main site of E2 synthesis [21]. Furthermore, INL neurons control photoreceptor homeostasis by sustaining structural and trophic support to the vasculature [22].

The CC demonstrated the highest vessel area, vessel density, average vessel length, vessel diameter index and tortuosity with the lowest lacunarity and the total number of endpoints. This is closely related to the high 
Table 2 Globe biometry normative values with gender distribution in four-year-old Caucasian children $(n=62)$

\begin{tabular}{|c|c|c|c|c|c|}
\hline \multirow{2}{*}{$\begin{array}{l}\text { Component } \\
\text { (measurement unit) }\end{array}$} & \multicolumn{2}{|c|}{ Male $(n=29)$} & \multicolumn{2}{|c|}{ Female $(n=33)$} & \multirow[t]{2}{*}{$P$ value ${ }^{*}$} \\
\hline & mean & SD & mean & SD & \\
\hline Age (months) & 50.03 & 3.81 & 50.61 & 3.75 & 0.56 \\
\hline $\mathrm{AL}(\mathrm{mm})$ & 22.29 & 0.56 & 22.05 & 0.48 & 0.08 \\
\hline $\mathrm{ACD}(\mathrm{mm})$ & 3.44 & 0.25 & 3.39 & 0.29 & 0.50 \\
\hline $\mathrm{LT}(\mathrm{mm})$ & 3.67 & 0.15 & 3.70 & 0.23 & 0.52 \\
\hline SE (D) & 43.27 & 1.29 & 43.59 & 1.13 & 0.31 \\
\hline K1 (D) & 42.88 & 1.25 & 43.16 & 1.09 & 0.35 \\
\hline K2 (D) & 43.68 & 1.36 & 44.01 & 1.22 & 0.31 \\
\hline $\mathrm{CCT}(\mu \mathrm{m})$ & 541.48 & 31.20 & 537.21 & 29.53 & 0.58 \\
\hline WTW (mm) & 12.30 & 0.39 & 12.28 & 0.36 & 0.85 \\
\hline $\operatorname{IOL}(\mathrm{D})^{* *}$ & 25.55 & 1.10 & 25.94 & 1.36 & 0.23 \\
\hline
\end{tabular}

$n$ number of participants, $A L$ axial length, $A C D$ anterior chamber depth, $L T$ lens thickness, $K 1$ flat corneal meridian, $K 2$ steep corneal meridian, $S E$ spherical equivalent, CCT central corneal thickness, WTW white-to-white, $I O L$ intraocular lens, $S D$ standard deviation

*Statistical significance was measured using Student's t-test for independent samples $(P<0.05)$

**Acrysoft IQ monofocal SN60WF (Alcon Laboratories Inc., Fort Worth, Texas, USA), A-constant 118.7 using SRK-T formula

metabolic burden of photoreceptors and the key role of choroidal circulation in delivering nutrients and oxygen to the outer retinal layers, as well as in keeping the steady temperature. In healthy individuals, sweptsource optical coherence tomography angiography (SSOCTA) of the vessel density is within the range of the morphometric results assessed with tissue microscopy [22]. During normal aging (range 6-100 years) the CC vessel density and capillary diameter decrease linearly from 0.75 and $9.8 \mu \mathrm{m}$ in the first decade to 0.41 and $6.5 \mu \mathrm{m}$ in the tenth decade, respectively [23]. In fouryear-old children, we measured a higher $\mathrm{CC}$ vessel density of $79.74 \%$ (Additional file 1: Table S1). Sugano et al. observed healthy individuals aged $27.9 \pm 5.8$ using SS-OCTA and AngioTool software and reported lacunarity of the choriocapillaris $0.016 \pm 0.001$ [24]. As a comparison, in this study, the CC mean lacunarity was $0.00564 \pm 0.01$. The highest tortuosity of CC supports the proposed thermoregulatory function of the choroid as coiling increases the surface area to volume ratio resulting in more effective heat dissipation.

To give a more thorough insight into the perifoveal capillary plexus formation, we observed FAZ separated across four vascular retinal slabs (Table 1), although it has been recommended that the FAZ size should be measured using an en face projection that includes all retinal plexuses [1], rather than separating the FAZ area across the layers [25]. In our study, the FAZ area varied across capillary plexuses, but these differences were not found to be sex-dependent (Table 1, Additional file 1: Table S1). The FAZ area was the narrowest in ICP (Additional file 1: Table S1), while DCP and SVP indicated no difference in the FAZ size (Additional file 1: Table S3). The explanation for these patterns can be found in different mechanisms governing the development of the foveal capillaries. First, the SVP layer sets the boundary defining the presumed foveal avascular area before the foveal depression begins to form [26]. Opposite to the peripheral retina, at the fovea, DVP forms prior to ICP by downward sprouting of SVP capillaries [27]. This sequence of events may be due to the increased metabolic needs of central photoreceptors [26]. The sprouting capillaries from DVP then ascend into IPL to form ICP. Thus, SVP and DCP anastomose first, in the early postnatal period on the margin of the avascular area, while ICP forms anastomosis later, on the foveal slope. The former could provide the reasoning for similarities in VDI between SVP and DCP (Additional file 1: Table S3). This temporal and spatial pattern of the retinal vasculature growth is related to the total number of blind end capillaries being the lowest at DCP and the highest at ICP (Table 1, Additional file 1: Table S3). Moreover, ICP had the highest JD (Table 1, Additional file 1: Table S3). The JD is a measure of sprouting activity and indicates higher angiogenesis [6]. Provis et al. demonstrated a spatial correspondence between the astrocyte-vascular ring and the foveal rim, postulating that blind-ending capillaries directed into the foveal region indicate the presence of inhibitory vascular factors that generate a "no-go" region at the immature fovea [26]. We speculate that ICP angiogenesis must be extensive as the layer ties in with DCP and SVP, but the process itself could be halted at the same time as for DCP and SVP, leaving the highest TNEP. As the constitution of the foveal depression and perifoveal plexuses development are interdependent events, JD and TNEP analysis at different time points may further clarify the period of the foveal maturation.

Even in adults, data of the FAZ area in ICP are scarce. Park JJ et al. observed that FAZ was qualitatively the smallest and best demarcated at ICP, however, quantitative measurements were not performed [28]. Given our results, it is evidenced that this feature is thus already modelled at the age of four.

From the age of eight onwards, the FAZ area observed at SVP only was smaller compared to our study group [1, 29]. Zhang et al. evaluated sex-related differences and a significantly larger FAZ area was found in girls [1]. Our results of the larger FAZ area indicate that at the age of four, foveal pit is still widening and shallowing due to retinal stretch as a major factor in cone packing [27]. In 
contrast to retinal stretching, pars plana growth adjusts for further posterior eye elongation afterward [30]. In adults, an annual increase in the FAZ area was reported $[28,31]$.

For full-term born preschool children, normative data on macular thickness are limited [32], while metrics of the concomitant macular microvascular network are even more scarce [23]. How the components of thickness and vascular network are further coupled has yet to be declared. The central macula was the thinnest $(253.82 \mu \mathrm{m})$. The macular thickness of superior $(338.03 \mu \mathrm{m})$, inferior $(334.32 \mu \mathrm{m})$, and nasal $(335.39 \mu \mathrm{m})$ parafoveal areas were similar, while temporal parafoveolar area was thinner $(324.63 \mu \mathrm{m})$. However, the distribution of macular thickness and its sexual differentiation did not reach statistical significance (Table 1, Additional file 1: Table S2). Compared with literature values for sixyear-old children, our results could indicate that after the age of four the axial length is further increasing, the inner retina is thinning and foveal depression is still deepening. In our study, the topographic retinal profile of the thinnest temporal and the thickest superior retinal inner segment observed at the age of 4 reached significance at the age of six [32]. Furthermore, sexual dimorphism of the central and inner macula thickness and central macular volume is not recorded before the age of six, with boys reaching a higher value [32]. Ethnic differences in macular thickness demonstrate significantly thicker retinas in East Asian compared to white 6-yearold children [32].

Our results indicate that at the age of 4, ocular components $\mathrm{AL}$ and corneal power are within the asymptotic phase of growth (Additional file 1: Table S4). The mean axial length in our study was $22.17 \pm 0.53 \mathrm{~mm}$, consistent with earlier reports [33-37]. A slow increase in central corneal thickness and ACD until 10 years of age was observed [35, 37]. Our results, measuring CCT $539.21 \pm 30.15 \mu \mathrm{m}$ compare favorably with earlier studies of similar age groups [36, 38], however, these published data are not within the emmetropic refractive range. In the Gutenberg Eye Study, mean CCT was $557.3 \pm 34.3 \mu \mathrm{m}$ in male and $551.6 \pm 35.2 \mu \mathrm{m}$ in female adult subjects (age range 35-74years) [39]. Mean ACD and LT in our study were $3.42 \pm 0.27 \mathrm{~mm}$ and $3.69 \pm 0.19 \mathrm{~mm}$, respectively. For this age group, we could not find corresponding values obtained with partial coherence laser interferometry in published literature. Compared to the adult Caucasians' values for ACD $3.87 \pm 0.35 \mathrm{~mm}$ [40], LT of $4.05 \pm 0.20 \mathrm{~mm}$ [41], and WTW $13.23 \pm 0.44$ [42], our results are largely distinctive (Additional file 1: Table S4). The present study identified that apart from the chorioretina, sex-based differences were not found for any other biometric ocular components. Sex-related differences emerge during school age with shorter axial length [42], shallower ACD [37, 38], and steeper corneal curvatures in girls $[43,44]$. However, other studies did not confirm dimorphism [45]. Given our results at the age of four, the eye has a power of $25.76 \pm 1.25 \mathrm{D}$.

Although a relatively large sample size of uniform age was enrolled, we did not perform a longitudinal study to assess temporal trends of macular maturation. Objective refraction was not addressed in this study; therefore, this bias may cause some data deformation, however, a high threshold of $\leq 0,1 \log$ MAR was set to define normative visual acuity in four-year-old children.

\section{Conclusions}

To the best of our knowledge, this is the first study that measured tortuosity across all layers. Although considered to be a more robust indicator of vascular function compared to vessel width, the nonexistence of a universally adopted mathematical definition of tortuosity precludes its use in large population studies and its relation to local and systemic diseases. We believe that this proposed quantitative vessel tortuosity index can be used in future studies to further define its diagnostic potential as the indicator of early retinal and systemic pathology since it is operator-independent and easy to calculate. Due to the simplicity of AngioTool analysis, morphometric parameters, namely lacunarity, could be used as a noninvasive valuable tool in defining early amblyopia [46].

This database acquired baseline information on physiological vascular parameters among normal healthy emmetropic children needed for assessment of macular microvascular architecture impairments, detection of early inflammation response as well as monitoring response to drug and surgical treatment.

Further studies are needed to reveal temporal trends of macular vascular network maturation and sexual dimorphism.

\section{Abbreviations}

ACD: Anterior chamber depth; AL: Axial length; AVL: Average vessel length; CC: Choriocapillaris; CCT: Central corneal thickness; CMT: Central macular thickness; CMV: Central macular volume; DCP: Deep capillary plexus; DVC: Deep vascular complex; E2: 17ß-estradiol; EA: Explant area; FA: Fluorescein angiography; FAZ: Foveal avascular zone; FoT: Foveolar thickness; ICG: Indocyanine green; ICP: Intermediate capillary plexus; INL: Inner nuclear layer; IPL: Inner plexiform layer; JD: Junctions density; K1: Corneal curvature in the flattest meridian; K2: Corneal curvature in the steepest meridian; L: Lacunarity; LT: Lens thickness; M: Magnification; NP: Number of pixels; NPM: Number of pixels per mm2; OCTA: Optical coherence tomography angiography; PCV: Parafoveolar superior macular volume; PIT: Parafoveolar inferior macular thickness; PIV: Parafoveolar inferior macular volume; PNT: Parafoveolar nasal macular thickness; PNV: Average parafoveolar nasal macular volume; PST: Parafoveolar superior macular thickness; PTT: Parafoveolar temporal macular thickness; PTV: Parafoveolar temporal macular volume; RPCP: Radial peripapillary capillary 
plexus; SS-OCTA: Swept-source optical coherence tomography angiography; SVC: Superficial vascular complex; SVP: Superficial vascular plexus; T: Tortuosity; TNEP: Total number of endpoints; TNJ: Total number of junctions; TVL: Total vessel length; VA: Vessels area; VD: Vessels density; VDI: Vessel diameter index; WTW: White-to-white.

\section{Supplementary Information}

The online version contains supplementary material available at https://doi. org/10.1186/s12886-021-02122-y.

Additional file 1: Table S1. Distribution of the vascular parameters and FAZ surface of different examined areas for all eyes in the study $(n=62)$. Table S2. Distribution of macular thickness and volume. Table S3. Posthoc analysis of the examined variables. Table S4. Globe biometry normative values in four-year-old Caucasian children $(n=62)$.

\section{Acknowledgements}

Not applicable.

\section{Authors' contributions}

Conception and design of the study: $\mathrm{DB}, \mathrm{MBj}, \mathrm{MB}$. Analysis and interpretation: $D B, M B j, M B, L M, A K, B K E$. Writing of the article: $D B, M B j, A K$; Critical revision of the article: $D B, M B j, M B, L M, A K, B K E$. Data collection: DB, MBj, AK, LM. Provision of patients: $D B, M B j, A K$. Statistical expertise: DB, MBj, LM. Literature search: $\mathrm{DB}, \mathrm{MBj}, \mathrm{MB}, \mathrm{LM}, \mathrm{AK}, \mathrm{BKE}$. Administrative support: DB, MBj, MB, LM, AK, BKE. All authors read and approved the final manuscript.

\section{Funding}

The authors did not receive support from any organization for the submitted work.

\section{Availability of data and materials}

The datasets generated and/or analyzed during the current study are not publicly available due to the extensive and large-scale datasheets, but are available from the corresponding author on reasonable request.

\section{Declarations}

\section{Ethics approval and consent to participate}

The study complied with the requirements of the Declaration of Helsinki, national requirements for the conduct of retrospective studies and received approval from the University Hospital "Sveti Duh" Research Ethics Committee (protocol identifier 01-4212/13), and the Ethics Committee of Josip Juraj Strossmayer University of Osijek, Faculty of Medicine in Osijek, Croatia (protocol identifier 2158-61-07-19-63). All legal guardians of the participants signed informed consent.

\section{Consent for publication}

Not applicable.

\section{Competing interests}

The authors declare that they have no competing interests.

\section{Author details}

${ }^{1}$ University Eye Department, University Hospital Centre Osijek, Osijek, Croatia. ${ }^{2}$ Faculty of Medicine, Josip Juraj Strossmayer University of Osijek, Osijek, Croatia. 'University Eye Department, University Hospital "Sveti Duh", Sveti Duh 64, 10000 Zagreb, Croatia. ${ }^{4}$ Faculty of Dental Medicine and Health Osijek, Josip Juraj Strossmayer University of Osijek, Osijek, Croatia.

Received: 11 March 2021 Accepted: 28 September 2021

Published online: 05 October 2021

\section{References}

1. Zhang Z, Huang X, Meng X, Chen T, Gu Y, Wu Y, et al. In vivo assessment of macula in eyes of healthy children 8 to 16 years old using optical coherence tomography angiography. Sci Rep. 2017;7:8936.

2. Falavarjani KG, lafe NA, Velez FG, Schwartz SD, Sadda SR, Sarraf D, et al Optical coherence tomography angiography of the fovea in children born preterm. Retina. 2017;37:2289-94.

3. Yilmaz I, Ocak OB, Yilmaz BS, Inal A, Gokyigit B, Taskapili M. Comparison of quantitative measurement of foveal avascular zone and macular vessel density in eyes of children with amblyopia and healthy controls: an optical coherence tomography angiography study. J AAPOS. 2017;21:224-8.

4. Ministry of Health of the Republic of Croatia. National Preventive Program for Early Amblyopia Detection - Protocol. 2018. https://zdravstvo.gov.hr/ UserDocsImages//2018\%20Natje\%C4\%8Daji//Nacionalni\%20prevent vni\%20program\%20ranog\%20otkrivanja\%20slabovidnosti\%202018.2028.\%20scan.pdf. Accessed 11 Mar 2021.

5. Bušić $M$, Bjeloš M, Petrovečki $M$, Kuzmanović Elabjer B, Bosnar D, Ramić S, et al. Zagreb amblyopia preschool screening study: near and distance visual acuity testing increase the diagnostic accuracy of screening for amblyopia. Croat Med J. 2016;57:29-41.

6. Zudaire E, Gambardella L, Kurcz C, Vermeren S. A computational tool for quantitative analysis of vascular networks. PLoS One. 2011;6:e27385.

7. Spaide RF, Klancnik JM Jr, Cooney MJ. Retinal vascular layers imaged by fluorescein angiography and optical coherence tomography angiography. JAMA Ophthalmol. 2015;133:45-50.

8. Zhao Z, Wen W, Jiang C, Lu Y. Changes in macular vasculature after uncomplicated phacoemulsification surgery: optical coherence tomography angiography study. J Cataract Refract Surg. 2018;44:453-8.

9. Hendrickson A, Possin D, Vajzovic L, Toth C. Histologic development of the human fovea from midgestation to maturity. Am J Ophthalmol. 2012;154:767-778.e2.

10. Vasavada V, Shah SK, Vasavada VA, Vasavada AR, Trivedi RH, Srivastava S, et al. Comparison of IOL power calculation formulae for pediatric eyes. Eye (Lond). 2016;30:1242-50.

11. Alabduljalii T, Westall C, Reginald A, Farsiu S, Chiu SJ, Arshavsky A, et al. Demonstration of anatomical development of the human macula within the first 5 years of life using handheld OCT. Int Ophthalmol. 2018;39:1533-42.

12. Springer A, Hendrickson A. Development of the primate area of high acuity. 2. Quantitative morphological changes associated with retinal and pars plana growth. Vis Neurosci. 2004;21:775-90.

13. Chan G, Balaratnasingam C, Yu P, Morgan W, McAllister I, Cringle S, et al. Quantitative morphometry of perifoveal capillary networks in the human retina. Invest Ophthalmol Vis Sci. 2012;53:5502.

14. Lin Y, Jiang H, Liu Y, Gameiro GR, Gregori G, Dong C, et al. Age-related alterations in retinal tissue perfusion and volumetric vessel density. Invest Ophthalmol Vis Sci. 2019;60:685-93.

15. Yu J, Jiang C, Wang X, Zhu L, Gu R, Xu H, et al. Macular perfusion in healthy Chinese: an optical coherence tomography angiogram study. Invest Ophthalmol Vis Sci. 2015;56:3212-7.

16. Kuehlewein L, Tepelus TC, An L, Durbin MK, Srinivas S, Sadda S. Noninvasive visualization and analysis of the human parafoveal capillary network using swept source OCT optical microangiography. Invest Ophthalmol Vis Sci. 2015;56:3984-8.

17. Tan CS, Lim LW, Chow VS, Chay IW, Tan S, Cheong KX, et al. Optical coherence tomography angiography evaluation of the parafoveal vasculature and its relationship with ocular factors. Invest Ophthalmol Vis Sci. 2015;57:224-34.

18. Ozawa GY, Bearse MA Jr, Harrison WW, Bronson-Chastain KW, Schneck ME, Barez S, et al. Differences in neuroretinal function between adult males and females. Optom Vis Sci. 2014;91:602-7.

19. Salyer D, Lund T, Fleming D, Lephart E, Horvath T. Sexual dimorphism and aromatase in the rat retina. Brain Res Dev Brain Res. 2001;126:131-6.

20. Miyamoto N, Mandai M, Takagi H, Suzuma I, Suzuma K, Koyama S, et al. Contrasting effect of estrogen on VEGF induction under different oxygen status and its role in murine ROP. Invest Ophthalmol Vis Sci. 2002;43:2007-14.

21. Cascio C, Deidda I, Russo D, Guarneri P. The estrogenic retina: the potential contribution to healthy aging and age-related neurodegenerative diseases of the retina. Steroids. 2015;103:31-41. 
22. Usui Y, Westenskow P, Kurihara T, Aguilar E, Sakimoto S, Paris LP, et al. Neurovascular crosstalk between interneurons and capillaries is required for vision. J Clin Invest. 2015;125:2335-46.

23. Ramrattan RS, van der Schaft TL, Mooy CM, de Bruijn WC, Mulder PG, de Jong PT. Morphometric analysis of Bruch's membrane, the choriocapillaris, and the choroid in aging. Invest Ophthalmol Vis Sci. 1994;35:2857-64.

24. Sugano Y, Sekiryu T, Furuta M, Tomita R, Shintake H, Maehara H, et al. Morphometrical evaluation of the choriocapillaris imaged by sweptsource optical coherence tomography angiography. Clin Ophthalmol. 2018;12:2267-76.

25. Rocholz R, Corvi F, Weichsel J, Schmidt S, Staurenghi G. OCT angiography (OCTA) in retinal diagnostics. In: Bille J, editor. High resolution imaging in microscopy and ophthalmology. New York: Springer; 2019. p. 135-60.

26. Provis JM, Sandercoe T, Hendrickson AE. Astrocytes and blood vessels define the foveal rim during primate retinal development. Invest Ophthalmol Vis Sci. 2000;41:2827-36.

27. Provis J. Development of the primate retinal vasculature. Prog Retin Eye Res. 2001;20:799-821.

28. Park JJ, Soetikno BT, Fawzi AA. Characterization of the middle capillary plexus using optical coherence tomography angiography in healthy and diabetic eyes. Retina. 2016;36:2039-50.

29. Campbell J, Zhang M, Hwang T, Bailey ST, Wilson DJ, Jia Y, et al. Detailed vascular anatomy of the human retina by projection-resolved optical coherence tomography angiography. Sci Rep. 2017;7:42201.

30. Wolsley CJ, Saunders KJ, Silvestri G, Anderson RA. Investigation of changes in the myopic retina using multifocal electroretinograms, optical coherence tomography and peripheral resolution acuity. Vis Res. 2008;48:1554-61.

31. Garrity ST, lafe NA, Phasukkijwatana N, Chen X, Sarraf D. Quantitative analysis of three distinct retinal capillary plexuses in healthy eyes using optical coherence tomography angiography. Invest Ophthalmol Vis Sci. 2017:58:5548-55.

32. Huynh SC, Wang XY, Rochtchina E, Mitchell P. Distribution of macular thickness by optical coherence tomography: findings from a population-based study of 6-year-old children. Invest Ophthalmol Vis Sci. 2006:47:2351-7.

33. Bhardwaj $\bigvee$, Rajeshbhai GP. Axial length, anterior chamber depth-a study in different age groups and refractive errors. J Clin Diagn Res. 2013;7:2211-2.

34. Bach A, Villegas VM, Gold AS, Shi W, Murray TG. Axial length development in children. Int J Ophthalmol. 2019;12:815-9.

35. Nelson L, Olitsky S. Harley's pediatric ophthalmology. 6th ed. Philadelphia: Wolters Kluwer; 2015.
36. Gul A, Caglar C, Cınal A, Yasar T, Kılıc A. Ocular biometry and central corneal thickness in children: a hospital-based study. Arq Bras Oftalmol. 2014;77:152-4

37. Costa AMC, Calixto N, Milhomens EG, Cronemberger S. Axial length, anterior chamber depth, lens thickness and horizontal corneal diameter in normal children. Invest Ophthalmol Vis Sci. 2005;46:671.

38. Pediatric Eye Disease Investigator Group, Bradfield YS, Melia BM, Repka MX, Kaminski BM, Davitt BV, et al. Central corneal thickness in children. Arch Ophthalmol. 2011;129:1132-8.

39. Hoffmann EM, Lamparter J, Mirshahi A, Elflein H, Hoehn R, Wolfram C, et al. Distribution of central corneal thickness and its association with ocular parameters in a large central European cohort: the Gutenberg health study. PLoS One. 2013:8:e66158.

40. Qin B, Tang M, Li Y, Zhang X, Chu R, Huang D. Anterior segment dimensions in Asian and Caucasian eyes measured by optical coherence tomography. Ophthalmic Surg Lasers Imaging. 2012;43:135-42.

41. Richdale K, Bullimore MA, Zadnik K. Lens thickness with age and accommodation by optical coherence tomography. Ophthalmic Physiol Opt. 2008;28:441-7.

42. Isenberg S. Physical and refractive characteristics of the eye at birth and during infancy. In: Isenberg SJ, editor. The eye in infancy. St. Louis: Mosby; 1994. p. 36-51.

43. Scheiman M, Gwiazda J, Zhang Q, Deng L, Fern K, Manny RE, et al. Longitudinal changes in corneal curvature and its relationship to axial length in the correction of myopia evaluation trial (COMET) cohort. J Optom. 2016;9:13-21.

44. Twelker JD, Mitchell GL, Messer DH, Bhakta R, Jones LA, Mutti DO, et al. Children's ocular components and age, gender, and ethnicity. Optom Vis Sci. 2009;86:918-35.

45. Iribarren R, Morgan IG, Chan YH, Lin X, Saw SM. Changes in lens power in Singapore Chinese children during refractive development. Invest Ophthalmol Vis Sci. 2012;53:5124-30.

46. Talu S, Vladutiu C, Popescu LA, Lupascu CA, Vesa SC, Talu SD. Fractal and lacunarity analysis of human retinal vessel arborization in normal and amblyopic eyes. HVM Bioflux. 2012;5:45-51.

\section{Publisher's Note}

Springer Nature remains neutral with regard to jurisdictional claims in published maps and institutional affiliations.
Ready to submit your research? Choose BMC and benefit from:

- fast, convenient online submission

- thorough peer review by experienced researchers in your field

- rapid publication on acceptance

- support for research data, including large and complex data types

- gold Open Access which fosters wider collaboration and increased citations

- maximum visibility for your research: over $100 \mathrm{M}$ website views per year

At BMC, research is always in progress.

Learn more biomedcentral.com/submissions 